\title{
Template-Based Synthesis of Aluminum Nitride Hollow Nanofibers Via Plasma-Enhanced Atomic Layer Deposition
}

\author{
Cagla Ozgit-Akgun, Fatma Kayaci, Inci Donmez, Tamer Uyar, ${ }^{\dagger}$ and Necmi Biyikli ${ }^{\dagger}$ \\ UNAM - Institute of Materials Science and Nanotechnology, Bilkent University, Ankara 06800, Turkey
}

\begin{abstract}
Aluminum nitride (AIN) hollow nanofibers were synthesized via plasma-enhanced atomic layer deposition using sacrificial electrospun polymeric nanofiber templates having different average fiber diameters $(\sim 70, \sim 330$, and $\sim 740 \mathrm{~nm})$. Depositions were carried out at $200^{\circ} \mathrm{C}$ using trimethylaluminum and ammonia precursors. AIN-coated nanofibers were calcined subsequently at $500^{\circ} \mathrm{C}$ for $2 \mathrm{~h}$ to remove the sacrificial polymeric nanofiber template. SEM studies have shown that there is a critical wall thickness value depending on the template's average fiber diameter for AIN hollow nanofibers to preserve their shapes after the template has been removed by calcination. Best morphologies were observed for AIN hollow nanofibers prepared by depositing 800 cycles (corresponding to $\sim 69 \mathrm{~nm}$ ) on nanofiber templates having $\sim 330 \mathrm{~nm}$ average fiber diameter. TEM images indicated uniform wall thicknesses of $\sim 65 \mathrm{~nm}$ along the fiber axes for samples prepared using templates having $\sim 70$ and $\sim 330 \mathrm{~nm}$ average fiber diameters. Synthesized AIN hollow nanofibers were polycrystalline with a hexagonal crystal structure as determined by high-resolution TEM and selected area electron diffraction. Chemical compositions of coated and calcined samples were studied using X-ray photoelectron spectroscopy (XPS). High-resolution XPS spectra confirmed the presence of AIN.
\end{abstract}

\section{Introduction}

I II-NITRIDE compound semiconductors (AlN, GaN, and InN) and their alloys have emerged as promising materials for a wide range of electronic device applications. Aluminum nitride (AIN), which is the widest band gap compound of III-nitride family, exhibits attractive material properties such as wide and direct band gap of $6.2 \mathrm{eV}$ (hexagonal AlN), small (even negative) electron affinity, significant piezoelectric response, good dielectric properties, chemical stability, high thermal conductivity, and low thermal expansion. Owing to this unique set of properties, nanostructures of AlN (e.g., nanotubes, ${ }^{1-6}$ hollow nanofibers, ${ }^{7}$ etc.) have recently attracted considerable attention as promising candidates for high surface area, high sensitivity chemical and biological sensor applications. ${ }^{8,9}$ Template-free or template-based approaches may be adopted for the syntheses of nanostructures. In most cases, template-free methods were

A. Bandyopadhyay — contributing editor

Manuscript No. 31889. Received August 09, 2012; approved September 13, 2012. Presented in part at the 2011 Fall Meeting of the Materials Research Society, Boston, MA, November 30, 2011 (Symposium BB, Poster No. BB15.5).

Presented in part at the 12th International Conference on Atomic Layer Deposition, Dresden, Germany, June 19, 2012 (Poster Session A, Poster No. 7).

Presented at the TechConnect World Nanotech Conference \& Expo 2012, Santa Clara, CA, June 20, 2012 (Exhibit and Poster Session II, Nanostructured Coatings, Surfaces \& Films).

Based in part on the thesis that will be submitted by C. Ozgit-Akgun for the Ph.D. degree in materials science and nanotechnology, Bilkent University, Ankara 2013, Turkey.

Author to whom correspondence should be addressed. e-mail: tamer@unam.bilkent. edu.tr and biyikli@unam.bilkent.edu.tr reported for tubular AIN nanostructures. Polycrystalline cubic AlN ( $c$-AlN) nanotubes were synthesized by gas-phase condensation using the solid-vapor equilibrium and their field emission properties were investigated. ${ }^{1}$ Faceted hexagonal AIN $(h-$ AlN) nanotubes were obtained by nitriding the aluminum powder in a horizontal tubular furnace. ${ }^{2} c$-AlN nanotubes were produced by reacting $\mathrm{AlCl}_{3}$ and $\mathrm{NH}_{3}$ gases at $1200^{\circ} \mathrm{C} .^{3} \mathrm{Na}-$ notubes of AlN were obtained as the side product while synthesizing a bulk layer of AlN polycrystals. ${ }^{4}$ Amorphous AlN nanotubes filled with nickel nanoparticles were also realized through the reaction of $\mathrm{NH}_{3}$ over $\mathrm{Ni}-\mathrm{Al}$ thin film at $1000^{\circ} \mathrm{C} .^{5}$ Recently, synthesis of $h$-AIN nanotubes by a roll-up approach at $350^{\circ} \mathrm{C}$ was reported, using $\mathrm{AlP}$ and $\mathrm{NaN}_{3}$ as the $\mathrm{Al}$ and $\mathrm{N}$ sources, respectively. ${ }^{6}$ Polycrystalline $h$-AIN hollow nanofibers were produced by carbothermal reduction and nitridation of precursor fibers obtained by electrospinning. ${ }^{7}$

Methods reported in the above-mentioned studies generally require high temperatures $\left(>1000^{\circ} \mathrm{C}\right)$ and in some cases there exist additional morphologies such as nanoparticles or nanowires in the final product. The most obvious constraint of template-free synthesis is probably the limited control over the properties of resulting structure (e.g., crystal structure, dimensions, etc.). Template-based synthesis, on the other hand, is a straightforward way of producing nanostructures with controlled properties, which in general requires a suitable deposition method and a sacrificial substrate having the desired geometry. Selected template material should not only be resistant to the growth ambient (temperature, pressure, gases), but should be able to disappear with a simple postdeposition treatment as well, unless it has a function in the final structure. Yin et al. ${ }^{10}$ reported on the synthesis of coaxial C-AlN-C composite nanotubes at $1600^{\circ} \mathrm{C}$ using a chemical substitution reaction in a controllable two-stage process using multiwalled carbon nanotubes as templates. Another example was demonstrated by Stan et al., ${ }^{11}$ in which epitaxial $h$-AIN shells were grown by metal-organic chemical vapor deposition at $1000^{\circ} \mathrm{C}$ around $\mathrm{GaN}$ nanowire templates. Templates were then removed by annealing at $1120^{\circ} \mathrm{C}$ under $\mathrm{H}_{2}$ atmosphere, leaving behind empty AlN shells.

Temperatures used for the synthesis of AIN nanostructures can be lowered considerably by alternating the deposition method. Atomic layer deposition (ALD) is a special type of low-temperature chemical vapor deposition, in which the substrate is exposed to two or more precursors in a sequential manner. Figure 1(a) is the schematic representation of an ALD cycle, which in general consists of four steps: (i) introduction of the metal-containing precursor, (ii) purge or evacuation, (iii) introduction of the second precursor, and (iv) purge or evacuation. As precursor molecules do not react with themselves, each pulse results in a surface saturated with a monolayer of that precursor. ${ }^{12}$ Besides being a low-temperature process, ALD also offers precise thickness control as well as excellent uniformity and conformality with this selflimiting growth mechanism. These unique characteristics make ALD a powerful technique for synthesizing nanostructures through template-based methods.

Variety of templates can be used as the deposition temperature decreases. For instance, polymers are promising 

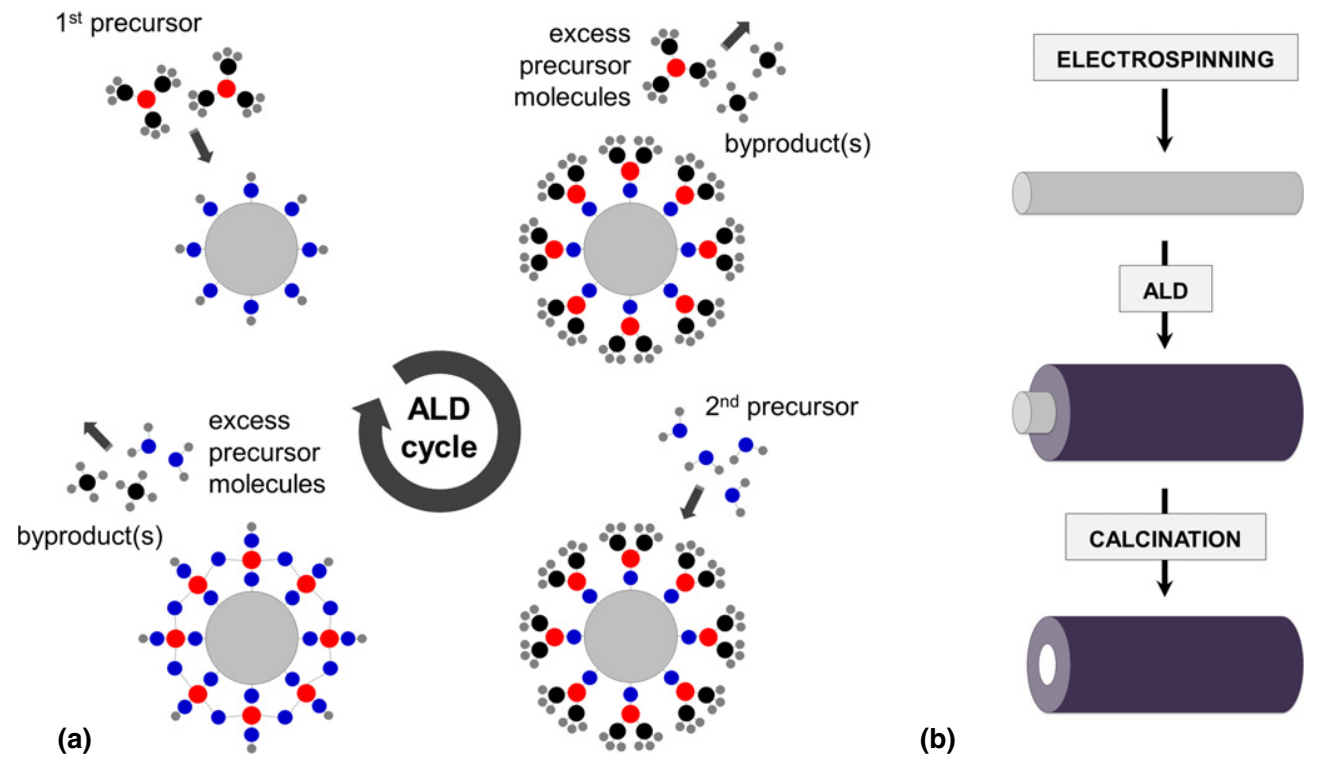

(b)

Fig. 1. (a) Schematic representation of an ALD cycle. (b) Template-based synthesis of inorganic hollow nanofibers; preparation of the nanofiber template by electrospinning, conformal deposition on electrospun nanofibers via atomic layer deposition, and removal of the organic template by calcination.

materials as sacrificial templates due to their availability, design flexibility, and very low cost. Polymeric fibers having diameters in the range of few micrometers to few hundred nanometers can be obtained via electrospinning, a basic process in which a polymer solution or melt pumped from syringe is subjected to high voltages. The shapes and dimensions of the electrospun nanofibers can be easily tuned by varying the polymer type, polymer concentration, solvent type, or controlling the electrospinning parameters including applied voltage, tip-to-collector distance, flow rate, etc. ${ }^{13}$ Very recently, electrospinning and ALD processes have been combined for synthesizing tubular nanostructures. Peng et $a l .{ }^{14}$ used electrospun polymeric fiber template for fabricating long and uniform metal-oxide microtubes with precise wall thickness control. In their study, $\mathrm{Al}_{2} \mathrm{O}_{3}$ was deposited by ALD on electrospun polymeric microfibers; polymeric core was then selectively removed by calcination. This approach was also applied for the fabrication of hollow nanofibers (or nanotubes) of various sizes from various materials such as $\mathrm{SnO}_{2},{ }^{15,16} \mathrm{TiO}_{2},{ }^{17,18}$ and $\mathrm{ZnO}^{19,20}$ using different electrospun polymeric nanofiber templates. Moreover, $\mathrm{NiFe}_{2} \mathrm{O}_{4}-\mathrm{TiO}_{2},{ }^{21} \mathrm{TiO}_{2}-\mathrm{ZnO},{ }^{22}$ and $\mathrm{SnO}_{2}-\mathrm{ZnO}^{23}$ core-shell nanofibers, as well as microtube-in-microtube $\mathrm{ZnAl}_{2} \mathrm{O}_{4}$ assemblies $^{24}$ were also fabricated successfully by combining electrospinning and ALD processes. To the best of our knowledge, synthesis of AIN hollow nanostructures by combining electrospinning and ALD has not yet been reported.

Here, we report on the template-based synthesis and characterization of AlN hollow nanofibers. The process has three steps [Fig. 1(b)]: (i) preparation of the Nylon 6,6 nanofiber template by electrospinning, (ii) conformal deposition of AIN on the electrospun polymer template via plasma-enhanced ALD (PEALD), and (iii) removal of the organic template by calcination.

\section{Experimental Procedure}

\section{(1) Electrospinning of Nylon 6,6}

Electrospun Nylon 6,6 nanofiber templates having different average fiber diameters were produced using different solvent systems [1,1,1,3,3,3-hexafluoro-2-propanol (HFIP; $\geq 99 \%$, Sigma-Aldrich, Chemie Gmbh, Munich, Germany) and formic acid (98\%-100\%; Sigma-Aldrich, Chemie Gmbh)] and polymer concentrations. 8 wt. \% Nylon 6,6 (relative viscosity 230.000-280.000) pellets were dissolved in formic acid; 5 wt.\% and 8 wt.\% Nylon 6,6 were dissolved in HFIP separately for $3 \mathrm{~h}$. Prepared homogeneous clear solutions were loaded individually in a $3 \mathrm{~mL}$ syringe fitted with a metallic needle having $0.8 \mathrm{~mm}$ inner diameter. Syringe was fixed horizontally on the syringe pump (Model: KDS 101; KD Scientific, Inc., Holliston, MA) and polymer solutions were pumped with a feed rate of $1 \mathrm{~mL} / \mathrm{h}$ during electrospinning. Electrospinning of the solutions was performed by applying a voltage of $15 \mathrm{kV}$ to the metal needle tip by high voltage power supply (AU Series; Matsusada, Precision Inc., Kusatsu-City, Shiga, Japan). Tip-to-collector distance was set at $10 \mathrm{~cm}$. On the way to the grounded stationary cylindrical metal collector (height: $15 \mathrm{~cm}$, diameter: $9 \mathrm{~cm}$ ) covered by a piece of aluminum foil, the solvents evaporated; and solid electrospun Nylon 6,6 nanofibers were deposited on the collector. The electrospinning setup was enclosed in a Plexiglas box, which allowed electrospinning process to be carried out at $24^{\circ} \mathrm{C}$ and $30 \%$ relative humidity. Morphologies and fiber diameters of the electrospun nanofibers were analyzed by SEM. Average fiber diameters of the samples were calculated by measuring diameters of $\sim 100$ different fibers from high magnification SEM images.

\section{(2) Synthesis of AlN Hollow Nanofibers}

AlN depositions were carried out at $200^{\circ} \mathrm{C}$ in Fiji F200-LL ALD reactor (Cambridge Nanotech, Inc., Cambridge, MA) with a base pressure of $30 \mathrm{~Pa}$. Four hundred and 800 cycles of AIN were deposited on electrospun Nylon 6,6 nanofibers via PEALD using trimethylaluminum (TMA) and ammonia $\left(\mathrm{NH}_{3}\right)$. One PEALD cycle consisted of $0.1 \mathrm{~s}$ TMA $/ 10 \mathrm{~s} \mathrm{Ar}$ purge $/ 40 \mathrm{~s} \mathrm{NH}_{3}$ plasma $(50 \mathrm{sccm}, 300 \mathrm{~W}) / 10 \mathrm{~s}$ Ar purge. Details of the recipe optimization are given elsewhere. ${ }^{25} \mathrm{Ar}$ was used as the carrier and purge gas. Precursor and plasma carrier gas flow rates were 60 and $200 \mathrm{sccm}$, respectively. In situ calcination of the AlN-coated nanofibers was carried out at $500^{\circ} \mathrm{C}$ for $2 \mathrm{~h}$ under $260 \mathrm{sccm}$ Ar flow. Samples were taken out from the reactor through a load-lock and exposed to air as soon as the ALD reactor cooled down to $200^{\circ} \mathrm{C}$. AlN hollow nanofibers were also prepared by ex situ calcination at air ambient $\left(500^{\circ} \mathrm{C}, 2 \mathrm{~h}\right)$.

\section{(3) Characterization Methods}

SEM and EDX studies were carried out using Quanta 200 FEG SEM (FEI, Hillsboro, OR) equipped with Ametek 
Apollo X silicon drift detector (EDAX Inc., Mahwah, NJ). Samples used for EDX were uncoated, whereas those used for SEM imaging were coated with $\sim 5 \mathrm{~nm} \mathrm{Au} / \mathrm{Pd}$ alloy. TEM and SAED analyses were performed using a Tecnai G2 F30 transmission electron microscope (FEI, Hillsboro, OR). TEM samples were prepared using two different approaches. In the first approach, polymer nanofibers were electrospun directly on copper grids, which were then coated with AlN and calcined in situ. Second approach consisted of putting a small piece of AIN hollow nanofiber network into a solvent (i.e., ethanol), followed by sonification to obtain individual hollow nanofibers dispersed through the solvent. AlN hollow nanofiber-containing solvent was then drop casted onto a copper grid and allowed to dry. Chemical composition and bonding states of the AlN nanostructures were investigated by X-ray photoelectron spectroscopy (XPS) using K-Alpha spectrometer (Thermo Fisher Scientific, Waltham, MA) with a monochromatized $\mathrm{Al} \mathrm{K} \alpha \mathrm{X}$-ray source.

\section{Results and Discussion}

Nylon 6,6 nanofiber templates having different average fiber diameters were obtained via electrospinning technique. Characteristics (composition and viscosity) of the Nylon 6,6 solutions, together with the morphologies and average fiber diameters of the electrospun Nylon 6,6 nanofibers are summarized in Table I. Type of the solvent used and concentration of polymer solution affected the polymer solution viscosity that is quite important for fiber diameters. Therefore, the viscosity of each Nylon 6,6 solution was different; accordingly, electrospinning of these solutions yielded Nylon 6,6 nanofibers with different fiber diameters. Less stretching of the electrified jet was occurred for more viscous polymer solutions and larger fiber diameters were obtained from these solutions, as it is anticipated. ${ }^{26,27}$ The representative SEM images of uniform and bead-free electrospun Nylon 6,6 nanofiber templates having smooth surfaces obtained from $8 \%(\mathrm{w} / \mathrm{v})$ formic acid, and $5 \%$ and $8 \%(\mathrm{w} / \mathrm{v})$ HFIP solutions are given in Fig. 2. Average fiber diameters of these Nylon 6,6 nanofibers were measured as $\sim 70, \sim 330$, and $\sim 740 \mathrm{~nm}$, respectively. These randomly oriented Nylon 6,6 nanofibers having different average fiber diameters were used as templates for the fabrication of AlN hollow nanofibers.

Four hundred cycles AlN were deposited on an electrospun template (average fiber diameter $\sim 740 \mathrm{~nm}$ ) at $200^{\circ} \mathrm{C}$ by PEALD. Recently, we have reported the deposition rate of AlN at this temperature as $0.86 \AA$ /cycle for planar substrates, ${ }^{25}$ which corresponds to a $\sim 34 \mathrm{~nm}$ thick film for 400 cycle deposition. As expected, the characteristic self-limiting growth mechanism resulted with highly uniform and conformal AIN layers on electrospun Nylon 6,6 nanofibers [Fig. 3(a)]. However, integrity of these conformal layers could not be retained after ex situ calcination at air ambient [Figs. 3(b) and (c)]. Although wall thickness of the resulting inorganic hollow nanofibers could easily be controlled by the number of ALD cycles, there seems to be a critical wall-

Table I. Properties of Nylon 6,6 Solutions and The Resulting Electrospun Nanofibers

\begin{tabular}{lcccc}
\hline $\begin{array}{l}\text { Solvent } \\
\text { system }\end{array}$ & $\begin{array}{c}\text { \% Nylon 6,6 } \\
(\mathrm{w} / \mathrm{v})\end{array}$ & $\begin{array}{c}\text { Viscosity } \\
(\mathrm{Pa} \mathrm{s})\end{array}$ & $\begin{array}{c}\text { Fiber } \\
\text { diameter } \\
(\mathrm{nm})\end{array}$ & $\begin{array}{c}\text { Fiber } \\
\text { morphology }\end{array}$ \\
\hline Formic acid & 8 & 0.0228 & $67 \pm 35$ & $\begin{array}{c}\text { Bead-free } \\
\text { nanofibers } \\
\text { Bead-free } \\
\text { nanofibers } \\
\text { Head-free } \\
\text { nanofibers }\end{array}$ \\
HFIP & 5 & 0.115 & $330 \pm 83$ & $737 \pm 266$ \\
\hline
\end{tabular}

${ }^{\dagger}$ With respect to the solvent.
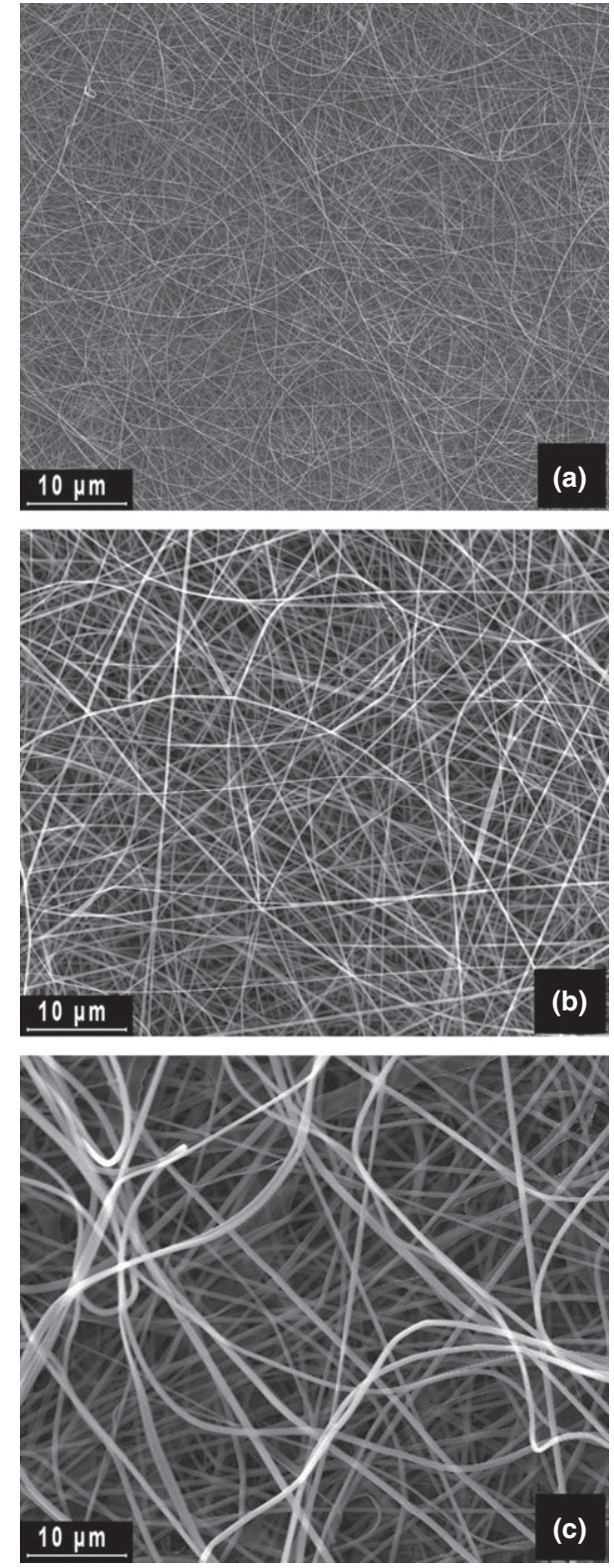

Fig. 2. SEM images of electrospun Nylon 6,6 nanofiber templates having (a) $\sim 70 \mathrm{~nm}$, (b) $\sim 330 \mathrm{~nm}$, and (c) $\sim 740 \mathrm{~nm}$ average fiber diameters.

thickness-to-inner-diameter ratio for ALD-grown layers to preserve their shapes after the sacrificial templates have been removed by calcination.

Figure 4(a) shows the SEM image of nanostructures synthesized by 800 -cycle ALD growth of AlN (corresponding to $\sim 69 \mathrm{~nm}$ AlN wall thickness) on $\sim 740 \mathrm{~nm}$ diameter fiber templates followed by an in situ heat treatment under continuous Ar flow. Resulting structures were hollow, although they have been calcined at an oxygen-free ambient. The critical wall-thickness-to-inner-diameter ratio could not be reached despite the doubled number of ALD cycles. Conformal AIN layer was unable to preserve the cylindrical geometry of the electrospun fiber template, which resulted in a hollow ribbon-like morphology. Figure 4(b) is the SEM image of hollow nanofibers synthesized by depositing 800 cycles AIN on a template having $\sim 330 \mathrm{~nm}$ average fiber diameter. For this combination, the resulting structure was an ideal replicate of the electrospun nanofiber template. EDX indicated the presence of $\mathrm{Al}, \mathrm{N}, \mathrm{O}$, and $\mathrm{C}$ in this sample. SEM image of hollow nanofibers synthesized by depositing 800 cycles AIN on a template having $\sim 70 \mathrm{~nm}$ average fiber diameter is given in Fig. 4(c). It is seen that individuality of the fibers has been 

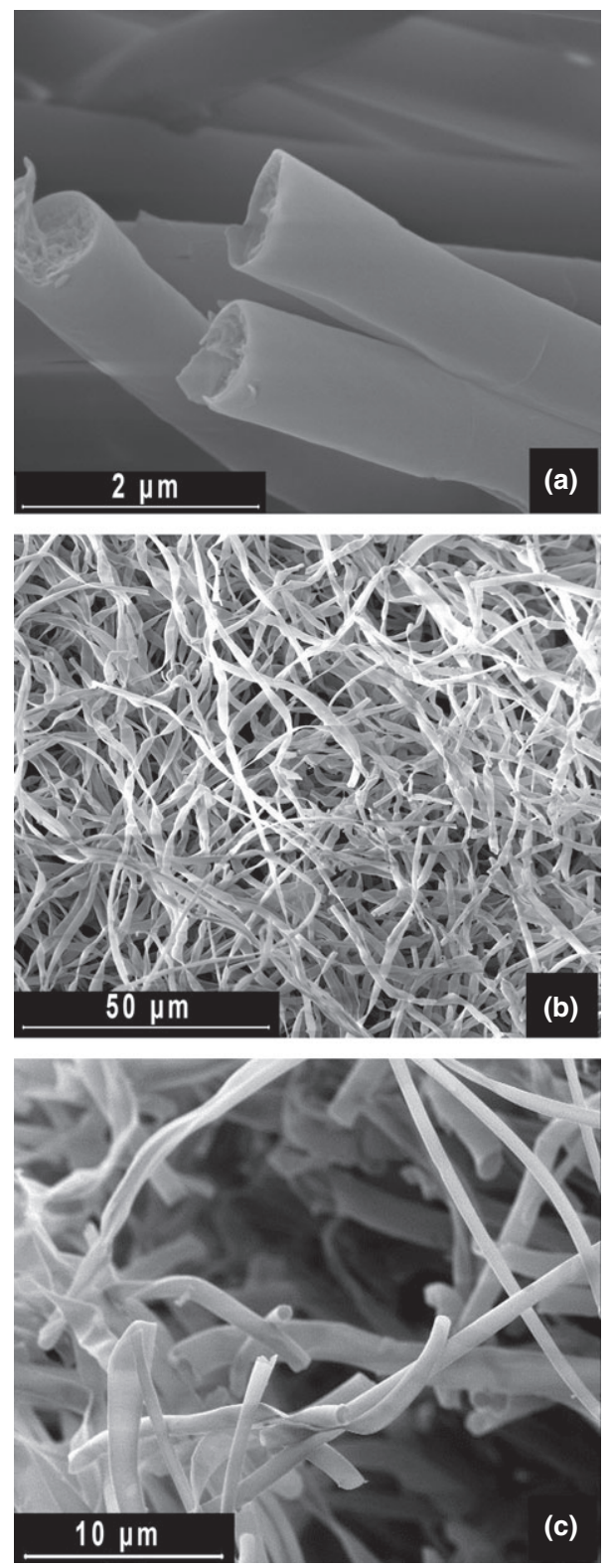

Fig. 3. SEM images of (a) Nylon 6,6 nanofibers (average fiber diameter $\sim 740 \mathrm{~nm}$ ) coated with 400 cycles $\mathrm{AlN}$, and (b, c) inorganic hollow nanofibers synthesized by the ex situ calcination of coated nanofibers.

lost due to the coalescence of AlN layers deposited on different fibers. The cross-sectional SEM image of this sample revealed that AlN fibers were hollow [inset of Fig. 4(c)], yet, the wall-thickness-to-inner-diameter ratio for this combination was too high.

Figure 5 belongs to AIN hollow nanofibers synthesized by the deposition of 800 cycles on a template having $\sim 330 \mathrm{~nm}$ average fiber diameter, followed by ex situ calcination. Each fiber was coated with a uniform and conformal layer of AlN. These layers preserved their shapes even after calcination at air ambient and resulted in a structure that is composed of continuous hollow nanofibers.

Bright field scanning TEM image of sample prepared by the deposition of 800 cycles on a template having $\sim 70 \mathrm{~nm}$ average fiber diameter, followed by in situ calcination is given in Fig. 6(a). It is seen that the wall thicknesses of hollow nanofibers are highly uniform along the fiber axes. Figures 6(b)-(d) are the TEM images of samples prepared using templates having average fiber diameters of $\sim 70, \sim 330$, and $\sim 740 \mathrm{~nm}$, respectively. Wall thicknesses of the AlN hol-
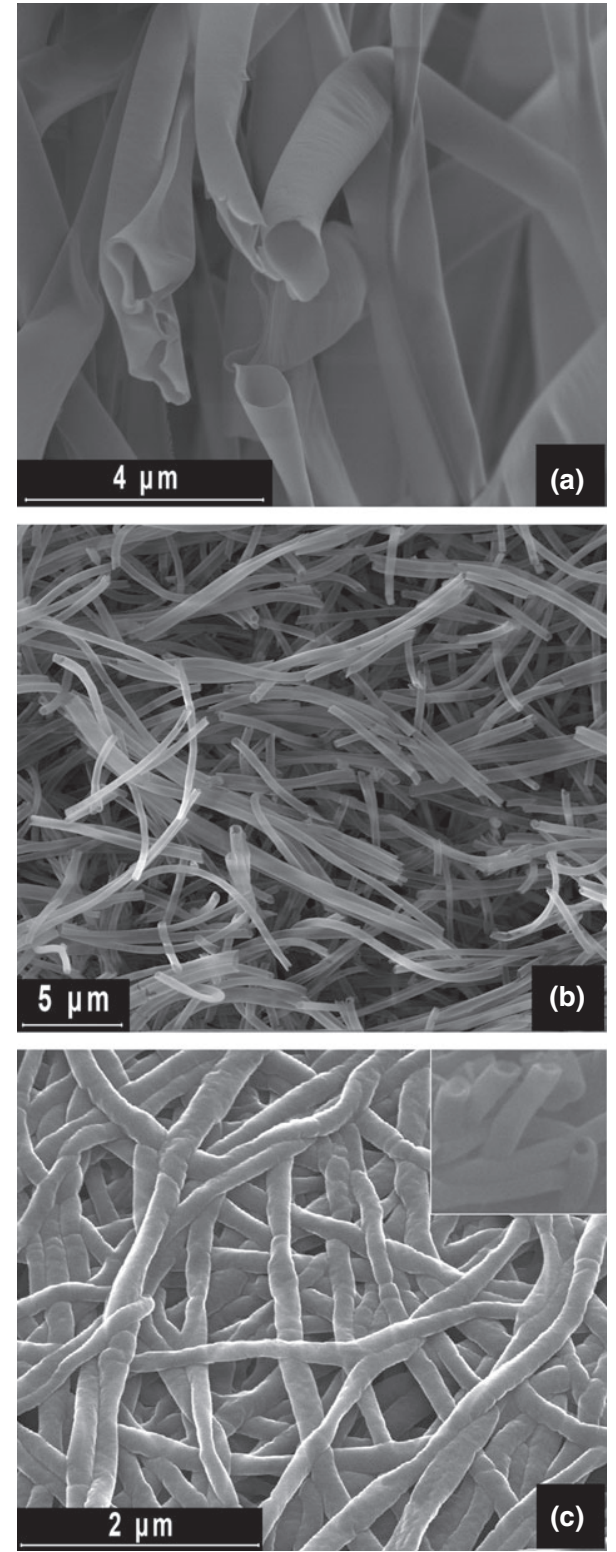

Fig. 4. SEM images of hollow nanofibers synthesized by depositing 800 cycles AlN on Nylon 6,6 templates having (a) $\sim 740 \mathrm{~nm}$, (b) $\sim 330 \mathrm{~nm}$, and (c) $\sim 70 \mathrm{~nm}$ average fiber diameters. AlN-coated fibers were calcined in situ.

low nanofibers shown in Figs. 6(b) and (c) were measured as $\sim 65 \mathrm{~nm}$, which is quite consistent with the deposition rate of AlN PEALD process at $200^{\circ} \mathrm{C}$. For the sample shown in Fig. 6(d), wall thickness was $\sim 32 \mathrm{~nm}$. This value is lower than the expected coating thickness, and might be related to the TEM sample preparation approach that was followed. For the samples shown in Figs. 6(a)-(c), few nanofibers were electrospun on copper grids, which were then coated and calcined in situ. These fibers were at the very top during the PEALD of AlN. For the sample shown in Fig. 6(d), on the other hand, TEM sample was prepared by sonification and drop casting. This individual nanofiber might therefore belong to any position along the out of plane direction. As AIN recipe has been optimized for planar substrates, the precursor doses used in this experiment may not be enough for coating such a high surface area sample. Even if the TMA and $\mathrm{NH}_{3}$ doses are sufficient for self-terminating reactions to take place at the fiber surface, there may have not been enough time for them to diffuse into the nanofiber matrix. 
Crystal structure of the AlN film deposited on Nylon 6,6 nanofiber templates was studied by high-resolution TEM (HR-TEM) and SAED. HR-TEM image of the AIN hollow nanofiber synthesized using $\sim 330 \mathrm{~nm}$ diameter Nylon 6,6 nanofibers is given in Fig. 6(e). AlN was found to be polycrystalline with nanometer-sized grains, which was further
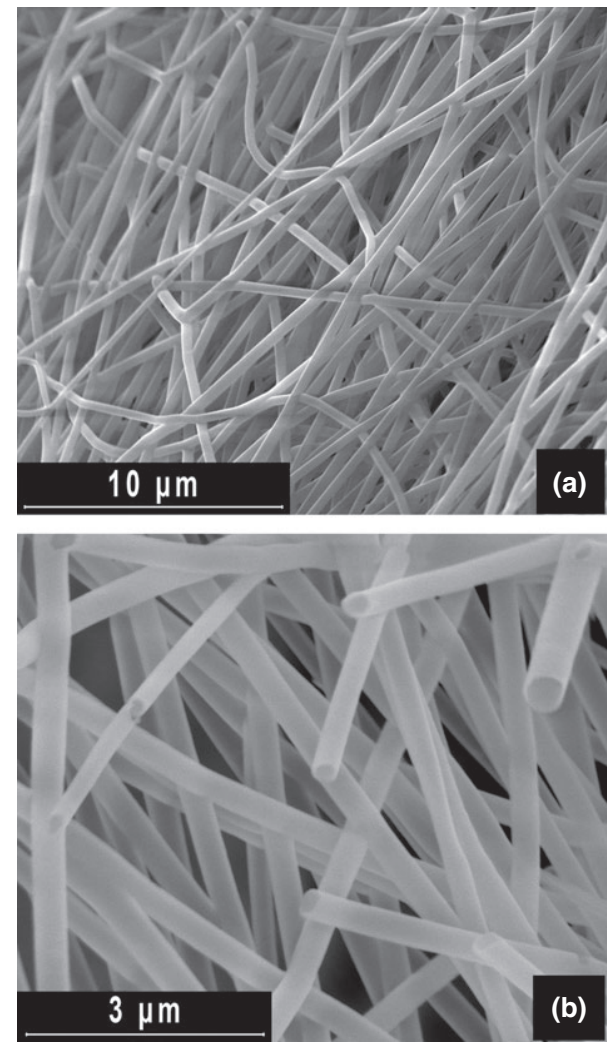

Fig. 5. SEM images of AlN hollow nanofibers taken at different magnifications. Hollow nanofibers were synthesized by the deposition of 800 cycles AIN on a Nylon 6,6 template having 330 $\mathrm{nm}$ average fiber diameter, followed by ex situ calcination at air ambient. confirmed by SAED. SAED pattern of the same sample [Fig. 6(e)] revealed seven polycrystalline diffraction rings, representing the $h$-AlN phase. Table II summarizes the SAED results, theoretical values for $h$-AlN, and corresponding planes. When same recipe was deposited on planar $\mathrm{Si}$ (100) substrates, the same seven reflections of the $h$-AlN phase appeared in the grazing-incidence X-ray diffraction pattern. ${ }^{25}$

Chemical compositions of bare and coated Nylon 6,6 nanofibers, as well as synthesized AlN hollow nanofibers were investigated by XPS, which is a surface analysis technique that collects data from the top $\sim 5-10 \mathrm{~nm}$ of the sample. In this study, the relatively large spot size $(\sim 400 \mu \mathrm{m})$ of the X-ray beam interacted with a large number of coated or calcined nanofibers, which might have discontinuities or cracks on the AlN shell due to the sample preparation procedure applied. Therefore, the collected data probably represent the organic content and Nylon 6,6-AlN interface in addition to the PEALD-grown AIN surface. Survey scans detected peaks of $\mathrm{Al}, \mathrm{N}, \mathrm{O}$, and $\mathrm{C}$ for coated and calcined samples prepared by depositing 800 cycles AIN on a template having $\sim 330 \mathrm{~nm}$ average fiber diameter (Table III). Ex situ calcination at $500^{\circ} \mathrm{C}$ for $2 \mathrm{~h}$ must have resulted in complete removal of the organic component. Accordingly, Al content increased and $\mathrm{N}$ content decreased when coated sample was calcined. An increased amount of $\mathrm{O}$ is believed to be due to the oxidation of AlN films upon annealing at air ambient. The 19.18 at.\% C present in the calcined sample, on the other hand, corresponds either to the surface contamination or calcination residues. XPS survey scan results of in situ calcined sample were quite similar to those of coated sample. There was no significant decrease in $\mathrm{C}$ concentration upon calcination. In other words, calcination at an oxygen-free ambient was not as effective as that performed at air ambient, although SEM images showed hollow structures for both cases. Results therefore indicate oxidation of AlN layer in the case of ex situ calcination and ineffectiveness of in situ calcination in terms of removing the organic component.

High-resolution XPS (HR-XPS) scans were also obtained to reveal bonding states of AlN hollow nanofibers synthesized at different conditions. Charging effects were corrected
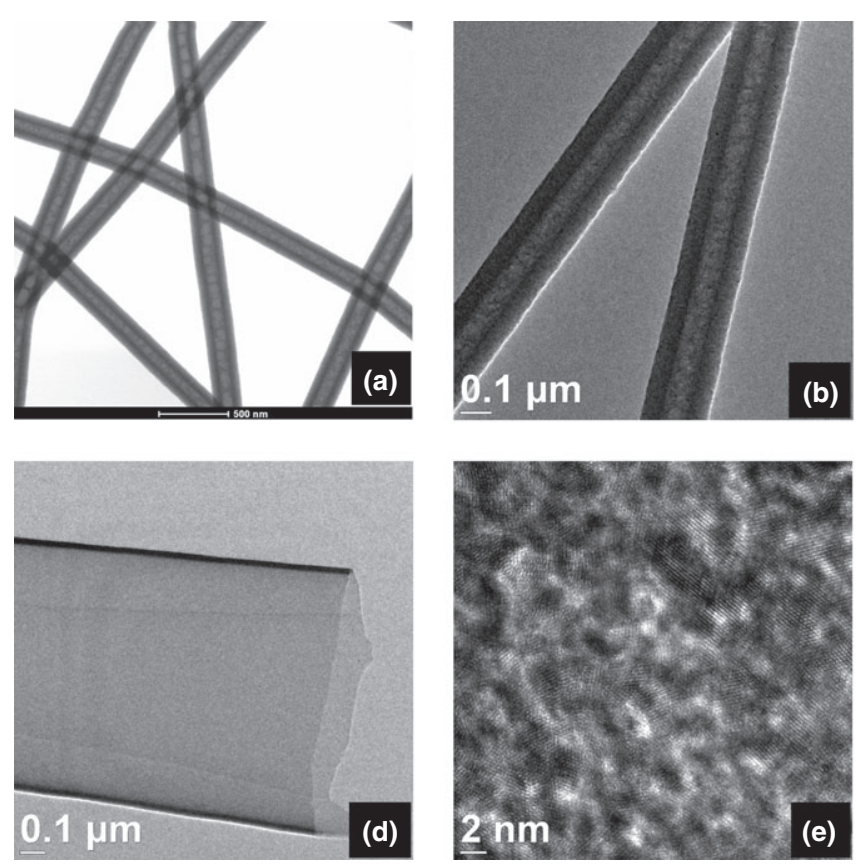
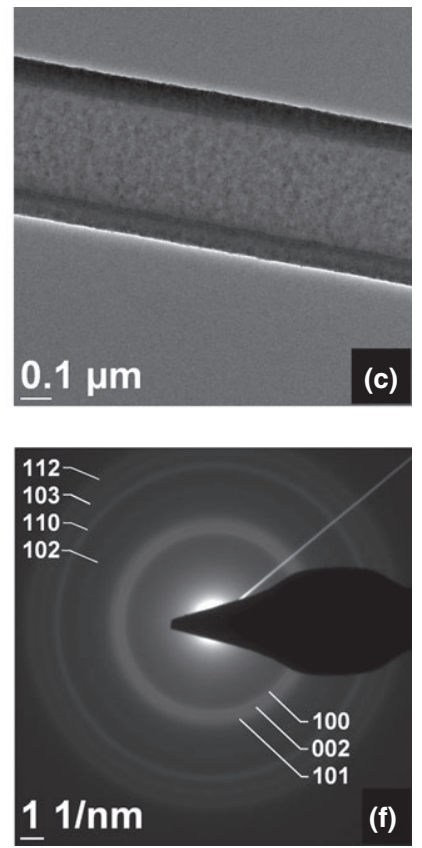

Fig. 6. AlN hollow nanofibers synthesized by the deposition of 800 cycles AlN, followed by in situ calcination. (a) Bright field TEM image of hollow nanofibers synthesized using Nylon 6,6 template having an average fiber diameter of $\sim 70 \mathrm{~nm}$. (b)-(d) TEM images of hollow nanofibers synthesized using templates with average fiber diameters of $\sim 70, \sim 330$, and $\sim 740 \mathrm{~nm}$, respectively. (e) High-resolution TEM image, and (f) SAED pattern of AlN hollow nanofibers synthesized using a template having $\sim 330 \mathrm{~nm}$ average fiber diameter. 
Table II. SAED Results, Theoretical Values, and Corresponding Crystallographic Planes

\begin{tabular}{llcc}
\hline \multirow{2}{*}{$\begin{array}{l}\text { Diameter } \\
(1 / \mathrm{nm})\end{array}$} & \multicolumn{2}{c}{ Interplanar spacing, $d(\AA)$} & \\
\cline { 2 - 3 } & Calculated & Theoretical $^{\dagger}$ & Corresponding plane, $h k l$ \\
\hline 7.434 & 2.690 & 2.6950 & 100 \\
8.057 & 2.482 & 2.4900 & 002 \\
8.451 & 2.367 & 2.3710 & 101 \\
11.036 & 1.8123 & 1.8290 & 102 \\
12.952 & 1.5442 & 1.5559 & 110 \\
14.199 & 1.4085 & 1.4133 & 103 \\
15.261 & 1.3105 & 1.3194 & 112 \\
\hline
\end{tabular}

${ }^{\dagger}$ Hexagonal AIN, ICDD reference code: 00-025-1133.

Table III. XPS Survey Scan Results. 800 Cycles AIN Were Deposited on a Template Having $\sim 330 \mathrm{~nm}$ Average Fiber Diameter

\begin{tabular}{lcccc}
\hline & \multicolumn{4}{c}{ Elemental composition (at.\%) } \\
\cline { 2 - 5 } Sample & $\mathrm{Al}$ & $\mathrm{N}$ & $\mathrm{O}$ & $\mathrm{C}$ \\
\hline Bare template & - & 9.79 & 11.71 & 78.5 \\
Coated template & 20.69 & 8.33 & 33.74 & 37.24 \\
After ex situ calcination & 33.02 & 5.78 & 42.02 & 19.18 \\
After in situ calcination & 21.87 & 7.51 & 37.14 & 33.49 \\
\hline
\end{tabular}

for coated and calcined samples using the adventitious carbon peak located at $285 \mathrm{eV}$. C 1s peak of the bare template having $\sim 70 \mathrm{~nm}$ average fiber diameter was fitted by multiple subpeaks, one of which (corresponding to the surface $\mathrm{C}$ contamination) was found to be located at $\sim 285 \mathrm{eV}$. No correction was therefore made for the bare template. Al 2p HR-XPS scans of hollow nanofibers synthesized by the in situ calcination of templates coated with 800 cycles AlN are shown in Fig. 7(a). As expected, Al 2p peaks were found to locate at the same position for samples synthesized using templates having different average fiber diameters (i.e., $\sim 70$ and $\sim 740 \mathrm{~nm}$ ). The data obtained from the sample prepared using $\sim 740 \mathrm{~nm}$ average fiber diameter template was fitted by two subpeaks located at 73.33 and $74.32 \mathrm{eV}$, corresponding to $\mathrm{Al}-\mathrm{N}^{28}$ and $\mathrm{Al}-\mathrm{O}^{29}$ bonds, respectively. The Al-O bond indicates surface oxidation, which might be expected as the samples were taken out from the ALD reactor and exposed to air at $200^{\circ} \mathrm{C}$. N 1s HR-XPS scans of the samples, as well as that of the bare template having an average fiber diameter of $\sim 70 \mathrm{~nm}$ are given in Fig. 7(b). $\mathrm{N} 1 \mathrm{~s}$ data obtained from the sample prepared using $\sim 740 \mathrm{~nm}$ average fiber diameter template were fitted by two subpeaks. The peak located at $396.57 \mathrm{eV}$ was attributed to the $\mathrm{N}-\mathrm{Al}$ bond $^{28}$; whereas the one located at $399.06 \mathrm{eV}$ was assigned as the N-O bond. ${ }^{30}$ The presence of the $\mathrm{N}-\mathrm{O}$ bond, which was also observed for the bare Nylon 6,6 template, is a direct proof of the existence of organic component in the sample prepared by in situ calcination. The peak representing the $\mathrm{N}-\mathrm{O}$ bond was also observed for the sample coated with 800 AlN cycles; however, it disappeared after ex situ calcination at air ambient. The temperatures required for the removal of sacrificial Nylon 6,6 nanofiber templates are not high and the whole process can be carried out inside the ALD reactor. As Nylon 6,6 nanofibers could not be completely removed through heat treatment at an oxygen-free ambient, the gas composition and gas flow rates must be adjusted accordingly during calcination.

Al 2p HR-XPS scans of coated and calcined (both in situ and ex situ) samples prepared using polymeric templates having $\sim 70 \mathrm{~nm}$ average fiber diameter were all fitted by two subpeaks located at $73.5 \pm 0.2(\mathrm{Al}-\mathrm{N}$ bond) and $74.5 \pm 0.3 \mathrm{eV}$
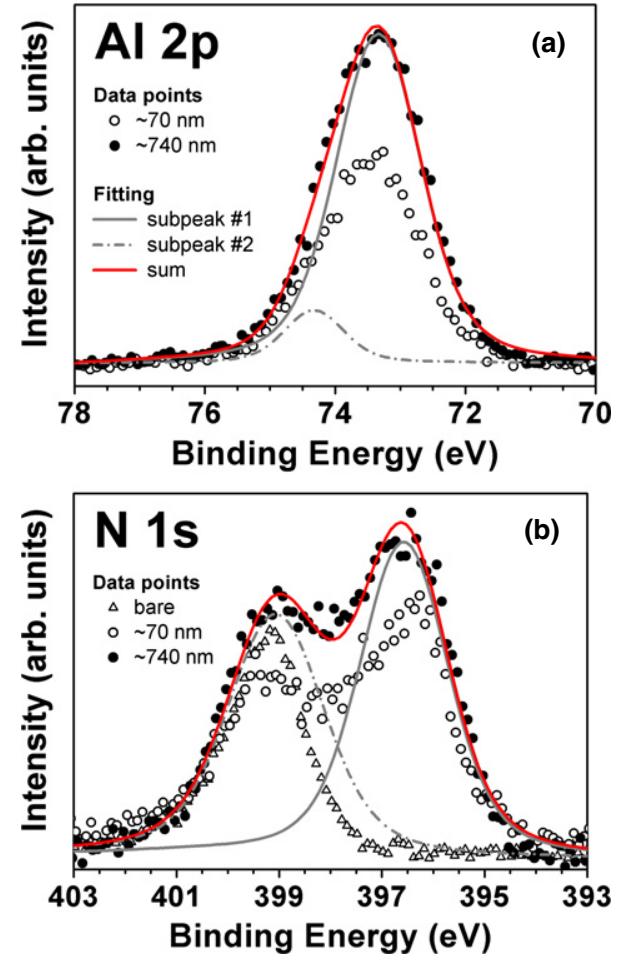

Fig. 7. (a) $\mathrm{Al} 2 \mathrm{p}$ and (b) $\mathrm{N}$ 1s high-resolution XPS scans of $\mathrm{AlN}$ hollow nanofibers synthesized by depositing 800 cycles AIN on Nylon 6,6 templates having $\sim 70$ and $\sim 740 \mathrm{~nm}$ average fiber diameters, which was followed by in situ calcination. $\mathrm{N}$ 1s highresolution XPS scan of bare Nylon 6,6 nanofiber template having an average fiber diameter of $\sim 70 \mathrm{~nm}$ is also included.

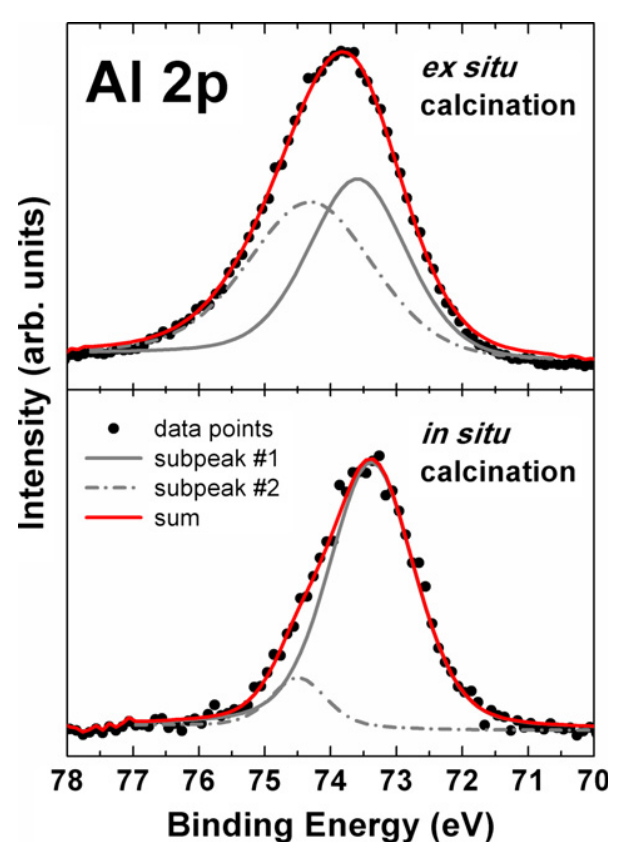

Fig. 8. Al 2p high-resolution XPS scans of AlN hollow nanofibers synthesized by the deposition of 800 cycles AlN on Nylon 6,6 nanofiber templates (average fiber diameter $\sim 70 \mathrm{~nm}$ ), followed by in situ and ex situ calcinations.

(Al-O bond). Figure 8 shows the Al 2p HR-XPS scans of calcined samples. When compared with that prepared by ex situ calcination, the sample prepared by in situ calcination exhibited a lower $\mathrm{Al}-\mathrm{O} / \mathrm{Al}-\mathrm{N}$ subpeak area ratio $(0.18$ vs $0.86)$ as expected. 


\section{Conclusions}

Template-based synthesis of AlN hollow nanofibers was demonstrated by combining the electrospinning and PEALD processes. Conformal AIN films were deposited at $200^{\circ} \mathrm{C}$ on sacrificial electrospun Nylon 6,6 nanofiber templates, using TMA and $\mathrm{NH}_{3}$ plasma. AlN-coated nanofibers were then calcined in situ or ex situ at $500^{\circ} \mathrm{C}$ for $2 \mathrm{~h}$ to remove organic components. SEM studies showed that there is a critical wall-thickness-to-inner-diameter ratio for AlN hollow nanofibers to preserve their shapes after the polymeric template has been removed by calcination. TEM images indicated uniform wall thicknesses along the fiber axes. Synthesized AlN hollow nanofibers were polycrystalline with a hexagonal crystal structure as determined by HR-TEM and SAED. Such high surface area AIN hollow nanofibers might potentially be used in high temperature ambient chemical sensing applications, where both high temperature compatibility and high sensitivity should meet.

\section{Acknowledgments}

This study was supported by the State Planning Organization (DPT) of Turkey through the National Nanotechnology Research Center (UNAM) Project. The authors acknowledge M. Guler from UNAM for TEM measurements. T. Uyar and N. Biyikli acknowledge Marie Curie International Reintegration Grant (IRG) for funding NANOWEB (PIRG06-GA-2009-256428) and NEMSmart (PIRG05-GA-2009-249196) projects, respectively. C. Ozgit-Akgun and F. Kayaci acknowledge TUBITAK-BIDEB for National PhD Scholarship.

\section{References}

${ }^{1}$ V. N. Tondare, C. Balasubramanian, S. V. Shende, D. S. Joag, V. P. Godbole, and S. V. Bhoraskar, "Field Emission from Open Ended Aluminum Nitride Nanotubes," Appl. Phys. Lett., 80 [25] 4813-5 (2002).

${ }^{2}$ Q. Wu, Z. Hu, X. Wang, Y. Lu, X. Chen, H. Xu, and Y. Chen, "Synthesis and Characterization of Faceted Hexagonal Aluminum Nitride Nanotubes," J. Am. Chem. Soc., 125 [34] 10176-7 (2003).

${ }^{3}$ L.-W. Yin, Y. Bando, Y.-C. Zhu, D. Golberg, and M. S. Li, "A Two-Stage Route to Coaxial Cubic-Aluminum-Nitride-Boron- Nitride Composite Nanotubes," Adv. Mater., 16 [11] 929-33 (2004)

${ }^{4}$ H. Morito, T. Ide, T. Karahashi, H. Orikasa, T. Yamada, and H. Yamane, "Synthesis of an AIN Polycrystalline Bulk Layer and Nanotubes by Using $\mathrm{NH}_{3}$ and Bi," J. Am. Ceram. Soc., 92 [11] 2578-82 (2009).

${ }^{5}$ R. Thapa, B. Saha, N. S. Das, U. N. Maiti, and K. K. Chattopadhyay, "Self Filling of Ni Nanoparticles in Amorphous AIN Nanotubes and its Field Emission Property," Appl. Surf. Sci., 256 [12] 3988-92 (2010).

${ }^{6} \mathrm{Y}$. Fan, "Formation of Crystalline AlN Nanotubes by a Roll-Up Approach," Mat. Lett., 65 [12] 1900-2 (2011).

${ }^{7}$ Y. Sun, J. Y. Li, Y. Tan, and L. Zhang, "Fabrication of Aluminum Nitride (AlN) Hollow Fibers by Carbothermal Reduction and Nitridation of Electrospun Precursor Fibers,” J. Alloy. Compd., 471 [1-2] 400-3 (2009).

${ }^{8}$ Z. Zhou, J. Zhao, Y. Chen, P. von Ragué Schleyer, and Z. Chen, "Energetics and Electronic Structures of AIN Nanotubes/Wires and Their Potential Application as Ammonia Sensors," Nanotechnology 18 [42] 424023 7pp (2007).

${ }^{9}$ A. Ahmadi, N. L. Hadipour, M. Kamfiroozi, and Z. Bagheri, "Theoretica Study of Aluminum Nitride Nanotubes for Chemical Sensing of Formaldehyde," Sensor. Actuat. B, 161 [1] 1025-9 (2012).

${ }^{10}$ L.-W. Yin, Y. Bando, Y. C. Zhu, M.-S. Li, C.-C. Tang, and D. Goldberg, "Single-Crystalline AIN Nanotubes with Carbon-Layer Coatings on the Outer and Inner Surfaces via a Multiwalled-Carbon-Nanotube-Template-Induced Route," Adv. Mater., 17 [2] 213-7 (2005).
${ }^{11}$ G. Stan, C. V. Ciobanu, T. P. Thayer, G. T. Wang, J. R. Creighton, K. P. Purushotham, L. A. Bendersky, and R. F. Cook, "Elastic Moduli of Faceted Aluminum Nitride Nanotubes Measured by Contact Resonance Atomic Force Microscopy," Nanotechnology 20 [3] 035706 6pp (2009).

${ }^{12}$ R. L. Puurunen, "Surface Chemistry of Atomic Layer Deposition: A Case Study for the Trimethylaluminum/Water Process," J. Appl. Phys. 97 [12] $12130152 \mathrm{pp}$ (2005).

${ }^{13}$ A. Greiner and J. H. Wendorff, "Electrospinning: A Fascinating Method for the Preparation of Ultrathin Fibers," Angew. Chem. Int. Ed., 46 [30] 5670-703 (2007).

${ }^{14}$ Q. Peng, X.-Y. Sun, J. C. Spagnola, G. K. Hyde, R. J. Spontak, and G. N. Parsons, "Atomic Layer Deposition on Electrospun Polymer Fibers as a Direct Route to $\mathrm{Al}_{2} \mathrm{O}_{3}$ Microtubes with Precise Wall Thickness Control," Nano Lett., 7 [3] 719-22 (2007).

${ }^{15}$ B.-S. Lee, W.-S. Kim, D.-H. Kim, H.-C. Kim, S.-H. Hong, and W.-R. $\mathrm{Yu}$, "Fabrication of $\mathrm{SnO}_{2}$ Nanotube Microyarn and Its Gas Sensing Behavior," Smart Mater. Struct. 20 [10] 105019 7pp (2011).

${ }^{16}$ W.-S. Kim, B.-S. Lee, D.-H. Kim, H.-C. Kim, W.-R. Yu, and S.-H. Hong, " $\mathrm{SnO}_{2}$ Nanotubes Fabricated Using Electrospinning and Atomic Layer Deposition and Their Gas Sensing Performance," Nanotechnology, 21 [24] 245605 $7 \mathrm{pp}$ (2010).

${ }^{7}$ G.-M. Kim, S.-M. Lee, G. H. Michler, H. Roggendorf, U. Gösele, and M. Knez, "Nanostructured Pure Anatase Titania Tubes Replicated from Electrospun Polymer Fiber Templates by Atomic Layer Deposition," Chem. Mater. 20 [9] 3085-91 (2008)

${ }^{8}$ S.-W. Choi, J. Y. Park, C. Lee, J. G. Lee, and S. Kim, "Synthesis of Highly Crystalline Hollow $\mathrm{TiO}_{2}$ Fibers Using Atomic Layer Deposition on Polymer Templates," J. Am. Ceram. Soc., 94 [7] 1974-7 (2011).

${ }^{19}$ J. Y. Park, S.-W. Choi, and S. S. Kim, "A Synthesis and Sensing Application of Hollow ZnO Nanofibers with Uniform Wall Thicknesses Grown Using Polymer Templates," Nanotechnology, 21 [47] 475601 9pp (2010).

${ }^{20}$ S. Cho, D.-H. Kim, B.-S. Lee, J. Jung, W.-R. Yu, S.-H. Hong, and S. Lee, "Ethanol Sensors Based on $\mathrm{ZnO}$ Nanotubes with Controllable Wall Thickness via Atomic Layer Deposition, an $\mathrm{O}_{2}$ Plasma Process and an Annealing Process," Sensor. Actuat. B, 162 [1] 300-6 (2012).

${ }^{21}$ E. Santala, M. Kemell, M. Leskelä, and M. Ritala, "The Preparation of Reusable Magnetic and Photocatalytic Composite Nanofibers by Electrospinning and Atomic Layer Deposition," Nanotechnology, 20 [3] 035602 5pp (2009).

${ }^{22}$ J. Y. Park, S.-W. Choi, J.-W. Lee, C. Lee, and S. S. Kim, "Synthesis and Gas Sensing Properties of $\mathrm{TiO}_{2}-\mathrm{ZnO}$ Core-Shell Nanofibers," J. Am. Ceram. Soc., 92 [11] 2551-4 (2009).

${ }^{23}$ S.-W. Choi, J. Y. Park, and S. S. Kim, "Synthesis of $\mathrm{SnO}_{2}-\mathrm{ZnO}$ CoreShell Nanofibers via a Novel Two-Step Process and Their Gas Sensing Properties," Nanotechnology, 20 [46] 465603 6pp (2009).

${ }^{24}$ Q. Peng, X.-Y. Sun, J. C. Spagnola, C. Saquing, S. A. Khan, R. J. Spontak, and G. N. Parsons, "Bi-directional Kirkendall Effect in Coaxial Microtube Nanolaminate Assemblies Fabricated by Atomic Layer Deposition," ACS Nano, 3 [3] 546-54 (2009).

${ }^{25}$ C. Ozgit, I. Donmez, M. Alevli, and N. Biyikli, "Self-Limiting Low-Temperature Growth of Crystalline AIN Thin Films by Plasma-Enhanced Atomic Layer Deposition," Thin Solid Films, 520 [7] 2750-5 (2012).

${ }^{26}$ Z. M. Huang, Y. Z. Zhang, M. Kotaki, and S. Ramakrishna, "A Review on Polymer Nanofibers by Electrospinning and Their Applications in Nanocomposites," Compos. Sci. Technol., 63 [15] 2223-53 (2003).

${ }^{27}$ T. Uyar and F. Besenbacher, "Electrospinning of Uniform Polystyrene Fibers: The Effect of Solvent Conductivity," Polymer, 49 [24] 5336-43 (2008).

${ }^{28}$ L. Rosenberger, R. Baird, E. McCullen, G. Auner, and G. Shreve, "XPS Analysis of Aluminum Nitride Films Deposited by Plasma Source Molecular Beam Epitaxy," Surf. Interface Anal., 40 [9] 1254-61 (2008).

${ }^{29}$ D. Manova, V. Dimitrova, W. Fukarek, and D. Karpuzov, "Investigation of D.C.-Reactive Magnetron-Sputtered AlN Thin Films by Electron Microprobe Analysis, X-Ray Photoelectron Spectroscopy and Polarised Infra-Red Reflection," Surf. Coat. Technol., 106 [2-3] 205-8 (1998).

${ }^{30}$ H. M. Liao, R. N. S. Sodhi, and T. W. Coyle, "Surface Composition of AIN Powders Studied by X-Ray Photoelectron Spectroscopy and Bremsstrahlung-Excited Auger Electron Spectroscopy," J. Vac. Sci. Technol., A, 11 [5] 2681-6 (1993). 